\title{
Retos y contradicciones de la formación de investigadores en México
}

\section{Desafios e contradições da formação de pesquisadores no México}

\section{Challenges and contradictions at the training the Researchers in Mexico}

\author{
Jorge Mario Flores Osorio*
}

\begin{abstract}
RESUMEN
En el artículo presento resultados de investigación con respecto al impacto que las políticas delineadas por el Estado mexicano, articuladas por el Consejo Nacional de Ciencia y Tecnología (CONACyT) e implementadas por las Instituciones de Educación Superior (IES), tienen en el proceso de formación de investigadores, con especial énfasis en los campos que corresponden a la educación y la psicología. El proceso de investigación se realiza a través de la observación participante, conversaciones con estudiantes y profesores de programas doctorales en los campos de la psicología y la educación y análisis de documentos oficiales en donde se postulan las políticas de investigación y las que refieren a la formación de investigadores en México. Analízanse las políticas planteadas por el Consejo Nacional de Ciencia y Tecnología (CONACyT) con relación a la función de la investigación en México, así como a las orientaciones que realiza a través del Programa Nacional de Posgrados de Calidad (PNPC) diseñado en la última década del siglo XX con la finalidad de garantizar la formación de investigadores con altos niveles de calidad. Aún aprecio el Programa para el Desarrollo Profesional Docente (PRODEP) en su pretensión de organizar a los investigadores en Cuerpos Académicos y garantizar, de esa manera, la productividad científica de los investigadores de las Instituciones de Educación Superior. Cuestiono las políticas del CONACyT con respecto a la formación de investigadores,
\end{abstract}

* Universidad de Tijuana. Programa de Posgrado (Doctorado) de Psicología y Educación. Tijuana, México. E-mail: jomafo@gmail.com. https://orcid.org/0000-0002-1024-1081. 
demostrando la inconsistencia de las mismas y su limitada aportación a la dinámica de calidad del posgrado de calidad.

Palabras clave: Contradicciones. Calidad formación. Investigación. Políticas.

\title{
RESUMO
}

No artigo, apresento os resultados da pesquisa sobre o impacto que as políticas delineadas pelo estado mexicano, articuladas pelo Conselho Nacional de Ciência e Tecnologia (CONACyT) e implementadas pelas Instituições de Ensino Superior (IES), têm no processo de formação de pesquisadores, com ênfase especial aos campos da educação e da psicologia. O processo de pesquisa é realizado por meio de observação participante, conversas com alunos e professores de doutorado nas áreas de psicologia e educação, além da análise de documentos oficiais onde são postuladas as políticas de pesquisa e aquelas que se referem à formação de pesquisadores no México. Neste artigo, analiso as políticas propostas pelo Conselho Nacional de Ciência e Tecnologia (CONACyT) em relação ao papel da pesquisa no México, bem como as diretrizes que propõem por meio do Programa Nacional de Pós-Graduação em Qualidade (PNPC) projetado na última década do século XX, a fim de garantir a formação de pesquisadores com altos níveis de qualidade. Da mesma forma, aprecio o Programa para o Desenvolvimento Profissional de Professores (PRODEP) em sua tentativa de organizar pesquisadores em Organismos Acadêmicos e garantir, assim, a produtividade científica dos pesquisadores das Instituições de Ensino Superior. Questiono as políticas do CONACyT quanto à formação de pesquisadores, demonstrando sua inconsistência e sua limitada contribuição para a dinâmica de qualidade da pós-graduação.

Palavras-chave: Contradições. Qualidade de formação. Pesquisa. Políticas.

\begin{abstract}
In the article I present research results regarding the impact, that the policies outlined by the Mexican State, articulated by the National Council of Science and Technology (CONACyT) and implemented by the Institutions of Higher Education (IES). they have in the process of training researchers, with special emphasis, in the fields that correspond to education and psychology. The research process is carried out through participant observation, conversations with students and professors of doctoral programs in the fields of psychology and education: besides the analysis of official documents where research policies are postulated and those that refer to the training of researchers in Mexico. In the communication I analyze the policies proposed by the
\end{abstract}


National Cluster of Science and Technology (CONACyT) in relation to the role of research in Mexico, as well as the guidelines that it carries out through the National Postgraduate Program in Quality (PNPC) designed in the last decade of the 20th century in order to guarantee the training of researchers with high levels of quality. In the same way, I value the Program for the Professional Development of Teachers (PRODEP) in its attempt to organize researchers in Academic Bodies and guarantee in this way, the scientific productivity of the researchers of Higher Education Institutions. I question CONACyT's policies regarding the training of researchers, demonstrating their inconsistency and their limited contribution to the quality dynamics of postgraduate quality.

Keywords: Contradictions. Quality training. Research. Policies.

\section{Introducción}

El presente artículo es resultado de la investigación que, en torno a las políticas de investigación y formación de investigadores en México, realizo desde hace cinco años y que desarrollo a partir de la observación participante y conversaciones con estudiantes de programas doctorales y con los profesores adscritos a ellos, aparte del análisis documental, especialmente, en el campo de la psicología y la educación.

En la comunicación muestro que, derivado del énfasis que las IES hacen en lo administrativo-contable, las aportaciones al conocimiento a través de la investigación quedan marginadas; además del poco interés que muestran por el desarrollo teórico-epistemológico en los egresados de los programas doctorales.

Evidencio las contradicciones que las políticas coordinadas por el Consejo Nacional de Ciencia y Tecnología (CONACyT) tienen con respecto a la realidad mexicana, también muestro que en los posgrados que forman investigadores no hay correspondencia con la necesidad mexicana de generar conocimiento pertinente.

En consecuencia, con las observaciones y el análisis de las conversaciones, evidencio que las visiones dogmáticas en torno a la investigación, inciden negativamente en la creatividad intelectual y la manera en la cual las políticas administrativo-contables limitan la Generación y Aplicación del Conocimiento (GAC), coherente con la realidad mexicana e indudablemente, su incidencia en la calidad de los egresados de dichos programas.

En la primera parte hago referencia al Consejo Nacional de Ciencia y Tecnología (CONACyT), a sus metas y objetivos, así como al Sistema Na- 
cional de Investigadores (SNI). Analizo las contradicciones manifiestas entre el discurso y la realidad mexicana. Posteriormente me refiero a las IES y su pretendida contribución al desarrollo del conocimiento y luego reflexiono en torno a las políticas del Programa para el Desarrollo Profesional Docente para el Tipo Superior (PRODEP). Finalmente, valoro la incidencia de las políticas promovidas por el CONACyT, la Asociación Nacional de Instituciones de Educación Superior (ANUIES) y la Subsecretaría de Educación Pública (SEP) en la formación de investigadores al interior de las IES.

\section{Consejo Nacional de Ciencia y Tecnología (CONACyT)}

El 29 de septiembre de 1970 se crea en México el CONACyT con la finalidad de generar políticas orientadas a desarrollar la Ciencia y la Tecnología, cubrir las demandas prioritarias de México, solucionar los problemas de la sociedad, además de contribuir a elevar el nivel de vida y el bienestar de la población. Sin embargo, a 57 años de la creación de dicha entidad los niveles de bienestar de la población mexicana son precarios. La tasa de empleo es menor que el promedio de la OCDE (62\%), la vivienda está por debajo del promedio, la esperanza de vida al nacer en promedio es de 75 años, cinco años menor al promedio de la OCDE; la tasa de homicidios en 2014 fue de 18 por cada 100 mil habitantes. Con respecto al apoyo social, México muestra las cifras más bajas, aparte de los bajos resultados en educación (OCDE, 2017).

El CONACyT (2012), según documento oficial, contribuye al incremento del potencial científico y tecnológico, eleva la calidad, la competitividad y la innovación de las empresas. Sin embargo, los indicadores muestran que solo el Estado de Nuevo León, tiene una relación significativa con el sector industrial (2014), ni siquiera la UNAM que está en el lugar número uno en casi todos los rubros de productividad científica y tecnológica, reporta relaciones concretas con la Iniciativa Privada (IP) y México no se encuentra entre los países con mayor aportación al conocimiento, menos del 1\%.

La misión del CONACyT (2012) para el año 2025, es fortalecer las actividades de investigación científica y tecnológica de calidad. El documento del CONACyT (2012) plantea que se contribuirá a enfrentar de forma pertinente los retos principales de la sociedad; además, reitera que busca elevar la calidad de vida de la población; a pesar de lo formulado en los documentos oficiales del CONACyT, 
Los niveles de pobreza monetaria en México han sido persistentes. Es preocupante constatar que la incidencia registrada en 2014 es de una magnitud similar a la que se observó en 1992 y que el número de personas en pobreza monetaria casi se ha duplicado en ese periodo (CORDERA; PROVENCIO, 2017, p. 67).

La desigualdad en México, con relación a los países que conforman la OCDE, es alta. La asimetría referida de pobres con respecto a los ricos es la más alta de los países que conforman dicho organismo (OCDE, 2017). Lo enunciado muestra que lo indicado en las políticas de creación y en la prospectiva del CONACyT a 2025 es inconsistente con la realidad mexicana.

Se postulan las siguientes áreas estratégicas de crecimiento: a) tecnologías de la información y la comunicación, b) biotecnología, c) materiales avanzados, d) diseño de procesos de manufactura, e) infraestructura y f) desarrollo urbano y rural. Lo indicado para el rubro f no se corresponde con los proyectos de investigación o con las tesis desarrolladas por los alumnos de posgrado en México.

Se afirma en las políticas del CONACyT que las innovaciones en las áreas referidas están orientadas a atender a la población menos favorecida, a las mujeres, a las personas con discapacidad, a los grupos indígenas y a los migrantes. Sin embargo, las convocatorias de investigación no están orientadas a los sectores de la población más desfavorecidos; por ejemplo, en la convocatoria del Programa de Estímulos a la Investigación, Desarrollo Tecnológico e Innovación, las líneas de trabajo son Innovación tecnológica para las Micro, Pequeñas y Medianas Empresas, Innovación para las Grandes Empresas (CONACyT, 2017) y en ningún momento se hace referencia al impacto en las condiciones de vida de los trabajadores o a los problemas de violencia generalizada que se vive en México, entre otros.

\section{Sistema Nacional de Investigadores (SNI)}

Por acuerdo presidencial el 26 de julio de 1984 se crea el Sistema Nacional de Investigadores en México, supuestamente para reconocer a quienes se dedican a la investigación científica y tecnológica. Pero la realidad fue que se tomó la decisión de crear dicho sistema a consecuencia del déficit fiscal del Estado mexicano, que no permitía ofrecer salarios dignos a los investigadores; de donde se constituyó en un parámetro de inclusión/exclusión con respecto a los financiamientos para la investigación. 
El SNI se convierte en espacio de competencia entre investigadores y el supuesto de reconocimiento como símbolo de calidad y prestigio en la aportación científica, resulta cuestionado, cuando se observan los niveles de simulación que se presentan en México con respecto a las publicaciones, las investigaciones y la formación doctoral (FLORES OSORIO; GARCÍA CEJUDO, 2006; FLORES OSORIO, 2016).

Se dice que el SNI promueve y fortalece la calidad a través de la evaluación de los productos de investigación científica y tecnológica, así como la innovación y contribuye a la formación y consolidación de investigadores de alto nivel, lo que supone, incrementa la cultura, la productividad, la competitividad y el bienestar del país, dicho que dista de la realidad mexicana, incluso en lo que se refiere a la formación de investigadores de alto nivel.

La incorporación de todas las entidades federativas mexicanas supondría un equilibrio en la producción de conocimiento a nivel nacional. Sin embargo, en la Ciudad de México se ubica el mayor número de investigadores y centros de investigación, aparte del Estado de Morelos, que es el segundo lugar en dicho rubro, pero no impacta en el desarrollo del Estado y menos en la solución a los problemas de pobreza, exclusión y violencia.

Con relación a la producción científica de las IES mexicanas, Zúñiga (2014) muestra que la Universidad Nacional Autónoma de México es la que más produce y que, a pesar de ubicarse en el primer lugar del ranking mexicano, a nivel mundial está en el $94^{\circ}$ y a nivel regional en el segundo, en ese mismo parámetro, la universidad que se encuentra en el lugar $12^{\circ}$, a nivel regional es el $64^{\circ}$ y a nivel mundial el $1657^{\circ}$, hecho que muestra que los objetivos de promover la investigación de calidad está muy lejos de cumplirse en México.

\section{IES y producción de conocimiento}

En las IES mexicanas los investigadores se debaten en un sinnúmero de contradicciones y procesos de inclusión/exclusión, por ejemplo, la pertenencia al SNI, el perfil deseable (real e ideal), la pertenencia a un Cuerpo Académico (en formación, consolidación o consolidado), a redes de investigación y a los mecanismos válidos para la obtención de financiamiento. En ese marco las IES 
tiene que poder traducirse a los términos puramente cuantitativos del equivalente universal del dinero, las instituciones terminan organizándose de tal modo que pueda producirse el mayor número de alumnos titulados y de artículos publicados... (PAVÓN, 2017, p. 11-12).

Los criterios postulados por el Estado a través de las instituciones coordinadoras de la investigación, se convierten en principios de imposición de lo relevante y lo necesario. Con ello, se elimina la creatividad de los investigadores y se anula el espíritu científico en los futuros investigadores e indudablemente aumenta la lucha por la obtención de recursos.

Las políticas ejecutadas por las IES se constituyen en mecanismos de control hacia los investigadores y niegan la complejidad de lo real, otorgan certeza dogmática a quienes se forman en los doctorados a través de ratificar los saberes adquiridos previamente y toda perspectiva que los cuestione es descalificada. Nunca se les enseña a los estudiantes a preguntar y menos a leer textos actualizados o como señala Pavón: “...la tarea más importante del profesor ya no es enseñar, sino evaluar, calificar, diplomar o titular, acelerar el proceso educativo y aumentar la eficiencia terminal, es decir, incrementar la producción de profesionistas a costa de la formación propiamente dicha..." (2017, p. 12).

Las instituciones que controlan los recursos para la investigación en México se tornan en espacios de poder y control del pensamiento universitario y a través de los fondos concursables se compran consciencias, es decir, que todo lo que se dice o se hace en las IES está controlado por el poder económico concentrado en instancias como el CONACyT o la SEP.

Los tiempos de culminación de una investigación se definen administrativamente y orillan a los investigadores, así como a los alumnos de posgrado, a realizar trabajos definidos por el tiempo y no por la solución de problemas, situación que lleva a los actores a considerar como investigación la aplicación y procesamiento de un instrumento que permite verificar lo ya sabido.

La imposición del pensamiento científico-positivo exige que los investigadores desarrollen su actividad en razón de la Investigación más Desarrollo (I+D), sugerido por Augusto Comte, en el ámbito de la Revolución Industrial y cuya finalidad se concreta a través del discurso de orden y progreso del mundo capitalista, que parece cobrar vigencia en el marco de la formación e investigación mexicana.

En el espacio del pensamiento capitalista, la investigación se constituye en principio del discurso cientificista universal, producido en los espacios eurocéntricos. Se niega la posibilidad de estudiar lo desconocido y los investigadores, 
...se encuentran inmersos en los vaivenes macro y micropolíticos, pues son los que movilizan los procesos llevados a cabo en la Universidad $\mathrm{y}$ son quienes pueden decidir si defienden el status quo conformista y, oportunistamente, se amoldan a él cínicamente o si procuran creativamente buscar otros mundos posibles (GONZÁLEZ CARDONA, 2016, p. 6).

La condición de cientificidad es asignada a lo que se concibe como método, por ejemplo, la experimentación, la estadística, la etnografía, la Investigación Acción Participativa, la hermenéutica y en el extremo los instrumentos como el Statical Package for the Social Sciencies (SPSS). La adopción dogmática de lo que pueda considerarse como método se convierte en un criterio de inclusión/ exclusión, de la misma manera que la selección de problemas supuestamente universales.

\section{Cuerpos académicos y políticas financieras}

Las políticas económico-financieras implementadas en México como complemento a los salarios académicos -estímulos al desempeño académico y beca del SNI- han generado fisuras irreparables en las dinámicas de investigación, así como en la estructura de los organismos gremiales de los trabajadores universitarios que se vuelven sumisos ante las autoridades, incluso avalan la corrupción hoy presente en las IES mexicanas.

Los lineamientos diseñados por el Estado Mexicano para controlar a las IES y a sus académicos se concretan a través de programas como PRODEP que impulsa la organización de Cuerpos Académicos (CA), que en realidad son agrupaciones circunstanciales en busca de los recursos ofertados por el Estado a través de la Secretaría de Educación Pública, además de mejorar el estatus al interior de las IES.

En las IES mexicanas los investigadores se mantienen alerta de las convocatorias institucionales para la investigación y los proyectos los hacen en función de lo que el Estado considera políticamente correcto. Realizan investigaciones discontinuas y acordes con las demandas de los países más desarrollados de la OCDE. Tales políticas convierten a los investigadores mexicanos en sectores subalternos y proveedores de información. Por ello, se priorizan las publicaciones en revistas indexadas en el Science Citation Index, en tal dimensión o SCOPUS. 
...se escribe cada vez más y se lee cada vez menos. O para enunciarlo de manera más precisa: los académicos deben dedicarse cada vez más a escribir, publicar y evidenciar publicaciones, lo que hace que tengan cada vez menos tiempo de leer, meditar lo que leen, reflexionar e incluso investigar (PAVÓN, 2017, p. 12).

El proceso de formación de los CA se realiza a través de afinidades personales, políticas o de conveniencia individual. En lo general, la conjunción no es mediada por la búsqueda de conocimiento pertinente sino por la perspectiva de aglutinarse para obtener recursos y simular estancias de investigación y participación en eventos académicos, en espera de una constancia que pueda canjearse por dinero, incluso simulan productividad académica.

Los CA se identifican con categorías que no se corresponden con líneas o programas de investigación, postulan objetivos que nunca se cumplen, puesto que al final se trabaja al margen de ejes claros de articulación científica, aunque con perspectivas semejantes con respecto a los recursos.

...se debe anotar que el conjunto de programas para otorgar tales reconocimientos a la labor de los grupos de profesionales que laboran en instituciones educativas de educación superior en México, se orientan a la labor individual del profesional y no al desempeño del grupo profesional. De esto es posible prever que el propio diseño de esas políticas y el alto grado de intervención del Estado en las comunidades de profesionales, al menos en este sector de la educación superior, ha limitado enormemente su autonomía y, en consecuencia, se han creado comunidades artificiales que han establecido contratos sociales que les permita operar de manera artificial como grupo, ya que se constituyen en la cobertura idónea para el desempeño altamente individualizado de sus funciones (BALLESTEROS, 2004, p. 4).

Los CA se identifican por el campo disciplinar de aplicación y nunca por una línea o programa de GAC. Por ejemplo, Psicología Educativa, cuya línea de generación y aplicación del conocimiento es el impacto de la tecnología moderna en los procesos educativos y psicológicos; Psicología Social, cuyas líneas son las variables psicosociales relacionadas con la salud y estudios sobre familia, pobreza, grupos vulnerables y exclusión social; Metodología de la Investigación o Economía de la Educación, la Ciencia y la Tecnología. 
Oficialmente, los CA se definen en tres momentos: consolidados, en consolidación y en formación, y se demarcan de acuerdo con los siguientes lineamientos.

1. Cuerpo Académico Consolidado: se define porque todos sus miembros tienen el grado de doctor y perfil preferente, hecho que supuestamente los habilita para la GAC y supone su potencial para formar a jóvenes investigadores.

2. Cuerpo Académicos en Consolidación: Suponen que un 50\% de sus miembros cuentan con el perfil preferente, así como productos sólidos de GAC, 33\% con experiencia en docencia y formación de recursos humanos, así como participación en redes.

3. Cuerpos Académicos en Formación: para grupos de académicos que no alcanzan los honores preferentes de la jerarquía institucional: $25 \%$ con perfil deseable y grado preferente, o en su defecto, $50 \%$ con perfil deseable.

Los lineamientos de PRODEP con relación a la calidad de los investigadores, es incongruente con la realidad universitaria, pues los profesores se han especializado en decir a los evaluadores lo que quieren escuchar, además de la simulación que existe con respecto a las publicaciones y al desarrollo de la investigación (FLORES OSORIO; GARCÍA CEJUDO, 2006).

Las políticas de PRODEP postulan la realización de actividades conjuntas entre los miembros del CA y con los alumnos en formación (publicaciones, investigaciones, participación en eventos académico-científicos, entre otros). Sin embargo, en la práctica se observa que las publicaciones y proyectos de investigación conjuntos, en realidad únicamente constituyen un acto formal de inclusión de los miembros del CA (FLORES OSORIO; GARCÍA CEJUDO, 2006), sin que realmente se participe de manera activa.

La lucha de los académicos por conquistar los espacios de poder provoca el desplazamiento o al menos el debilitamiento- de los investigadores que se colocan al otro lado del discurso institucional, de esa manera, los colectivos de investigación, denominados Cuerpos Académicos (CA), se constituyen, en clubes de la alabanza mutua y como señala Pavón “... puede apreciarse con facilidad en el ámbito académico a través de la vacuidad, la insignificancia y las tendencias tautológicas de las publicaciones...” (2017, p. 13).

Con respecto a la infraestructura con que cuentan los CA, si bien es cierto que pueden adquirir equipos y material para el desarrollo de su actividad, los recursos para instalación se otorgan de manera preferente a quienes se incorporan por primera vez a las IES, se niega tal posibilidad a profesores de tiempo completo que, por su permanencia en la IES, supuestamente, ya no necesitan 
los apoyos para adquisición y equipamiento de sus espacios de acción, no así para financiar el trabajo de investigación.

En síntesis, los CA no constituyen grupos colegiados de investigación preparados para la GAC pertinente con la realidad mexicana y menos con calidad, son agrupaciones circunstanciales y temporales conformadas para buscar financiamiento y acercarse de alguna manera a las líneas de poder y privilegio que las dinámicas de acreditación, certificación y credencialización permiten.

Por su lado, las políticas propuestas por el Estado mexicano, además de constituirse en obstáculo epistemológico, funcionan como estrategia de fragmentación de los académicos vinculados con las IES y se prioriza “... la forma sobre el contenido, el método sobre la teoría, el proceso evaluativo sobre la cosa evaluada, lo aparente sobre lo verdadero, los diplomas a costa de los aprendizajes" (PAVÓN, 2017, p. 12).

\section{El mito de la formación de investigadores}

Las IES mexicanas diseñan los programas de posgrado por los recursos que el CONACyT a través del PNPC ofrece, en consecuencia, los criterios de selección están centrados en principios cuantitativos, número de profesores reconocidos en el SNI, eficiencia terminal al margen de la calidad de las tesis y de los propios egresados, situación que: “... en términos más concretos, que no solo se lea cada vez menos, sino que se lean cada vez menos libros que artículos y cada vez menos artículos que resúmenes" (PAVÓN, 2017, p. 12-13).

En el diseño curricular se indica que el programa estará en consonancia con la Sociedad del Conocimiento (SC) o Economía del Conocimiento (EC) como la denomina el Banco Mundial (BM); sin embargo, lo dicho no coincide con la realidad. Al revisar los planes de estudio para formar doctores, observo inconsistencias con respecto a los objetivos, el perfil de egreso, los seminarios y los contenidos programáticos que no se corresponden con los nombres de los seminarios, incluso, se incorpora a doctores al margen de la pertinencia de su formación; es decir, sin considerar el perfil que cada seminario demanda.

El control institucional de los investigadores y de los programas de posgrado se consigue a través de una compleja telaraña que atrapa y condiciona las problemáticas de investigación y bajo esa dinámica, los profesores, afirman dogmáticamente que el método constituye un criterio de certeza para la investigación y orientan los trabajos de tesis al margen del trabajo de campo concentrando 
a los alumnos en los cubículos (oficinas de los profesores), especialmente en la investigación que se realiza en los campos de la psicología y la educación.

En el panorama referido, la investigación se convierte en hábito, costumbre y reiteración de tradiciones añejas, no necesariamente generadas por los científicos, dogmas que son reforzados y reproducidos en el proceso de formación doctoral a través de comités tutoriales, exámenes pre-doctorales, presentación de avances y prescripción de tiempos financieros para la conclusión de la tesis.

En los claustros de posgrado la reflexión epistemológica es negada, bajo el supuesto de que las estrategias (métodos y técnicas), legitimadas, garantizan la producción de conocimiento; sin embargo, se olvidan quienes conforman la planta docente, que los denominados métodos -verificacionista, falsación- fueron creados como criterios de demarcación por Filósofos de la Ciencia como Moritz Schlick, Karl Popper, Karl Hempel en su afán de demarcar la ciencia de la metafísica o la ciencia de la no ciencia.

La dinámica de formación de investigadores en México es un correlato dogmático de las prácticas de investigación hegemónicas y la actividad de los investigadores se reduce a la gestión de recursos y al seguimiento de reglas apegadas a marcos disciplinares, que legitiman, ciertos problemas de investigación cómodos para el status quo.

En las políticas de formación, el método, se convierte en varita mágica para resolver problemas de diversa índole. Con ello, se niega la pertinencia y relevancia de los problemas, además de su pertenencia a condiciones concretas de existencia y grado de desarrollo socioeconómico de cada país, hecho que requiere originalidad y claro posicionamiento en el espacio de producción científica. En el trayecto de la formación de investigadores, el método se convierte en obstáculo epistemológico y limitación para reconocer las estructuras concretas de la realidad.

El horizonte de investigación y formación de investigadores de las IES mexicanas niega la importancia que tiene la crítica en la producción de teorías, situación que se fortalece con instituciones hegemónicas como Consejo Mexicano de Investigación Educativa, Academia Mexicana de la Ciencia, Sociedad Mexicana de Psicología Social, Asociación Mexicana de Psicología Social, Consejo Nacional de Ciencia y Tecnología, entre otras. Por consecuencia, se niega la propia historia de la ciencia que muestra cómo el conocimiento es producto del análisis crítico de las teorías que pierden fuerza para explicar los problemas emergentes.

Bajo las pretensiones del Estado Mexicano de equilibrar el número de doctores con los países de la OCDE, se impulsa el PNPC como mecanismo de control y su conversión en instrumento para ideologizar a los jóvenes investigadores, en términos, de lo políticamente correcto de la investigación. Por lo que 
la originalidad de las tesis es reprimida por los tutores del alumno, convirtiendo a los egresados en amplificadores del pensamiento eurocéntrico.

La pretensión de las políticas que subyacen al PNPC con relación a la oferta de posgrado (maestría y doctorado) de calidad, tanto en lo que refiere a la formación profesionalizante como de investigación, es loable. Pero la concreción de dichas intenciones no se produce tal y como muestro en las seis observaciones que a continuación enumero.

Primera observación: pertenecer al PNPC tiene poco significado en términos de la oferta de posgrado de calidad, realmente significa una opción para que las IES obtengan reconocimiento. Pero realmente sirven para conseguir apoyos económicos, tanto para los profesores como becas para los estudiantes y la ubicación en el ranking y publicitar a las IES en el marcado de alumnos.

Segunda observación: un número significativo de aspirantes se acercan a los programas incorporados al PNPC, por la beca que se ofrece y que funge como sustituto de la limitada oferta de empleos y sus deprimentes salarios. En los casos de profesores de las IES que deciden estudiar un posgrado, la principal motivación es la beca que se convierte en complemento salarial y posibilidad de mejorar el salario al culminar el posgrado. Tercera observación: en los programas vinculados a la educación, los profesores son incorporados por el único hecho de contar con el grado de doctor. No importa la experiencia y es frecuente que, en razón del control del poder, se luche porque un alumno incondicional se integre a la planta docente, incluso, se le asignen estudiantes para dirección de tesis.

Cuarta observación: en razón de los criterios para mantenerse en el PNPC y el énfasis en la eficiencia terminal de los doctorandos, aunado a la necesidad de créditos que los profesores tienen para mantener sus estímulos o su ascenso en el SNI, se acelera el proceso de culminación de la tesis en detrimento de la calidad del documento (FLORES OSORIO; GARCÍA CEJUDO, 2006).

Quinta observación: los problemas de investigación que se formulan, además de ser irrelevantes para la sociedad mexicana, son poco originales e indudablemente su significado en términos de aportación al conocimiento es equivalente a cero.

Sexta observación: quien dirige la tesis, por lo general impone al alumno la problemática que a su juicio es relevante; además de priorizar el método por encima del problema y utilizar adjetivos para calificar el método o también orillar al alumno a la aplicación de instrumentos, en ocasiones ya diseñados o realizados para poblaciones limitadas que impiden la generación de conocimiento. 
Séptima observación: la pertenencia de los profesores al SNI no garantice la calidad de la investigación o de las publicaciones a las que aluden para someterse a evaluación; incluso, como recomendación a los alumnos se les indica que deben publicar incluyendo a su Cuerpo Tutorial o su director de tesis (FLORES OSORIO, 2016).

\section{Conclusiones}

La investigación y la Formación de investigadores en México se desarrolla bajo parámetros administrativo-contables postulados por el Estado mexicano a través de organismos como el Consejo Nacional de Ciencia y Tecnología (CONACyT), la Secretaría de Educación Pública (SEP) y por la Asociación Nacional de Universidades e Instituciones de Educación Superior (ANUIES).

Las políticas administrativo-contables que el Estado mexicano impone a las Instituciones de Educación Superior (IES) constituyen un mecanismo de intervención en los procesos de formación y en la generación y aplicación del conocimiento, de donde, los investigadores deben centrar su trabajo en lo que se considera políticamente correcto y no en la generación de conocimiento original.

La premura con la que se lleva a un estudiante de posgrado a culminar su investigación que se convertirá en tesis, incide negativamente en la calidad del producto e indudablemente en el aprendizaje del doctorando de los procesos de investigación efectivamente científica.

En las ocasiones los alumnos cumplen la función de proveedores de textos para los investigadores, y están motivados por la necesidad de aparecer en una publicación, aceptan hacerlo como coautores, a pesar de ser quienes efectivamente escriben los artículos, incluso, los profesores recomiendan a sus alumnos los incluyan en sus publicaciones y en ocasiones los obligan a que también los citen. Existen programas en los que la inclusión del Comité Tutoral en las publicaciones de los alumnos constituye un lineamiento que deben cumplir los doctorandos.

La formación de investigadores bajo parámetros de simulación no contribuye a resolver los problemas cruciales de la sociedad mexicana, tales como la violencia, la pobreza y el desempleo. Considero que para que la investigación científica y la innovación tecnológica contribuyan al desarrollo de la sociedad mexicana es indispensable que, además de aumentar el porcentaje de inversión en ese ámbito, el Estado respete la autonomía de los investigadores con respecto a la elección de problemas de investigación y a la posibilidad de construir las estrategias pertinentes para ellos. 


\section{REFERENCIAS}

BALLESTEROS, A. Cuerpos académicos y clausura profesional en la UPN. Campus Milenio, Suplemento Universitario, en Red de Investigadores sobre Educación Superior, n. 95, p. 4, 2004. Disponible en: <http://www.riseu.unam.mx/documentos/archivo/ Campus_Milenio/A.Ballestero1.pdf $>$.

CONSEJO NACIONAL DE CIENCIA Y TECNOLOGÍA. Breve historia del CONACyT. Disponible en: $<$ http://2006-2012.conacyt.gob.mx/Acerca/Paginas/default.aspx $>$. Aceso en: 2012.

CORDERA, R.; PROVENCIO, E. Propuestas estrategias para el desarrollo 2019-2024 (Avance). Ciudad de México, México: Universidad Autónoma de México, 2014.

DUTRÉNIT, G.; ZARAGOZA, M. L.; ZÚÑIGA-BELLO, P. Taller sobre indicadores en ciencia y tecnología en Latinoamérica. OCDE: FCCyT, 2014. Disponible en: $<$ http:// www.oecd.org/statistics/how-s-life-23089679.htm>. Aceso en: 2014.

FLORES OSORIO, J. M. Supuestos y realidades de la formación de investigadores educativos. Revista Altamira, Universidad de Tijuana CUT, Año 3, n. 14, p. 38-59, 2016.

FLORES OSORIO, J. M.; GARCÍA CEJUDO, M. Universidad pública en la sociedad global. En: FLORES OSORIO, J. M. (Coord.), Historia, modernidad y crisis de la educación superior. Ciudad de México, México, p. 85-110, 2006.

FORO CONSULTIVO CIENTIFICO Y TECNOLÓGICO A.C. Ranking nacional de ciencia, tecnología e innovación. Ciudad de México, México. FCCyT, 2004.

OCDE. Estudios económicos de la OCDE. 2017. Disponible en: <https://www.oecd. org/eco/surveys/mexico-2017-OECD-Estudios-economicos-de-la-ocde-vision-general. pdf>. Aceso en: 2017.

PAVÓN, D. Capitalismo y psicología en la historia latinoamericana: esbozo de recapitulación histórica para proyectos liberadores. En: PAVÓN, D. (Coord.), Capitalismo y psicología crítica en Latinoamérica. Del sometimiento neocolonial a la emancipación de subjetividades emergentes. Ciudad de México, México: Editorial Kanankil, 2017. p. 17-26.

Texto recibido en 17 de mayo de 2018. Texto aprobado en 17 de julio de 2018. 